CBIE-LACLO 2015

Anais do XXVI Simpósio Brasileiro de Informática na Educação (SBIE 2015)

\title{
WebMonitor: uma ferramenta para monitoramento e acompanhamento de cursos em um AVA
}

\author{
Jath da S. e Silva ${ }^{1}$, Ketlen K. Teles Lucena ${ }^{1,2}$, Elaine H. Teixeira de Oliveira ${ }^{1}$ \\ ${ }^{1}$ Instituto de Computação - Universidade Federal do Amazonas (UFAM) \\ Manaus, Brasil \\ ${ }^{2}$ Centro de Educação a Distância - Universidade Federal do Amazonas (UFAM) \\ Manaus, Brasil \\ \{jathsilva, ketlen.teles\}@gmail.com, elaine@icomp.ufam.edu.br
}

\begin{abstract}
It is really important to manage students in Learning Management Systems to reduce dropout rates and failure in semipresential or distant courses. In order to assist educational support in LMS, this paper presents WebMonitor, a plugin developed for Moodle. This application aims to facilitate the monitoring of academic activities by using graphic techniques of information visualization for the analysis of interactions of students with learning objects in the virtual classroom. In addition to supporting mediation, WebMonitor also allows students to self-assess themselves according to their commitment in course activities.
\end{abstract}

Resumo. Gerenciar o desempenho de alunos em um ambiente virtual de aprendizagem (AVA) é de fundamental importância para a redução dos índices de evasão e reprovação em cursos na forma semipresencial ou à distância. A fim de auxiliar o acompanhamento pedagógico em um AVA, este artigo apresenta o WebMonitor, um plugin desenvolvido para o AVA Moodle. Este aplicativo visa facilitar a tarefa de monitoramento de atividades acadêmicas utilizando técnicas gráficas de visualização da informação para a análise das interações dos estudantes com os elementos de aprendizagem da sala de aula virtual. Além de apoiar a mediação, o WebMonitor também permite que aluno se auto-avalie em relação ao seu comprometimento nas atividades do curso.

\section{Introdução}

Os Ambientes Virtuais de Aprendizagem (AVA) são ferramentas desenvolvidas especialmente para o gerenciamento de cursos via Web, possibilitando a realização de tarefas diversas, viabilizando o uso de vários recursos de âmbitos tecnológicos e pedagógicos. Os atuais AVAs são softwares capazes de gerenciar o conteúdo de disciplinas e manipular arquivos e mídias de diversos formatos, assim como controlar os registros de acesso, avaliações e notas [Pereira 2007].

Entretanto, ainda se encontram dificuldades para se acompanhar o desempenho acadêmicos de alunos em cursos oferecidos de forma virtual em um AVA. Os índices de reprovação, evasão e desistência são relativamente altos [Oliveira et al. 2012] e cada vez mais a necessidade de enfrentamento dessa realidade tem levado muitos pesquisadores da área educacional a conduzirem trabalhos no sentido de delinear as causas desses problemas. Pois, se por um lado as tecnologias permitem a oferta de cursos em demanda por outro, é preciso criar meios de acompanhar o andamento dos mesmos, diagnosticar com antecedência alunos com dificuldades, seja tecnológica ou de aprendizado, e assim 
traçar e executar formas de intervenção a fimm de garantir um máximo aproveitamento dos recursos disponíveis no AVA.

Com intuito de oferecer uma ferramenta de monitoramento de desempenho em um AVA, neste trabalho é apresentado o WebMonitor, que consiste em um plugin desenvolvido para o AVA Moodle. Esta ferramenta busca auxiliar os responsáveis acadêmicos no acompanhamento da participação de alunos em atividades como postagem de arquivos e interação em fóruns de discussão. Estes dados serão exibidos conforme solicitação do usuário a partir do acesso ao aplicativo. A análise desta informação mostra um perfil detalhado da performance da turma no ambiente virtual e dá a professores e mediadores condições de identificar possíveis desistências, reprovações ou evasão de alunos. Foi usada ainda uma ferramenta gráfica de visualização de informações conhecida como Treemap [Johnson, 1991].

A estrutura deste artigo está assim organizada: A Seção 2 trata de uma breve revisão da literatura sobre monitoramento e acompanhamento acadêmico em um AVA (subseção 2.1) e, na subseção 2.2 uma visão geral das técnicas de visualização da informação. A Seção 3 apresenta a arquitetura e funcionalidades do WebMonitor. Na Seção 4 é descrito um estudo de caso onde os experimentos com o sistema são realizados. A seção 5 analisa os principais resultados com estes experimentos. As conclusões são discutidas na Seção 6.

\section{Revisão da Literatura}

O levantamento de trabalhos relacionados com esta pesquisa desdobrou-se em duas frentes: (i) o acompanhamento e monitoramento de estudantes em AVAs e (ii) a aplicação de técnicas atuais de visualização de informação para auxiliar ferramentas como o WebMonitor na apresentação visual e na análise de dados.

\subsection{Monitoramento em um AVA}

Diversos trabalhos na literatura têm sido direcionados ao acompanhamento de ações de mediação e tutoria em cursos gerenciados por um AVA, semipresenciais e à distância, e muitos tem apresentado resultados consistentes. Em seguida, são citadas algumas pesquisas que contribuíram para o levantamento bibliográfico deste trabalho.

Trabalhos como o de Ferreira e Elia (2013), podem ser consultados para uma melhor compreensão das causas do fenômeno da evasão por meio da construção de uma rede sistêmica, onde são rastreados e agrupados em categorias todos os possíveis motivos destes problemas. Dentre as causas levantadas no estudo, as dificuldades no uso da tecnologia ou relacionados à falta de interação com a figura do professor-tutor aparecem em todas as categorias.

Conforme a pesquisa de Almeida et al. (2012), "Os principais LMS em uso no Brasil carecem de ferramentas que possibilitem o monitoramento ágil das ações de tutoria pelos gestores e pelo próprio tutor. ". No trabalho, são apresentados indicadores que fornecem dados de apoio ao acompanhamento do processo de ensino-aprendizagem aos mediadores de cursos à distância.

O trabalho de Reis e Barrére (2014) desenvolveu uma arquitetura para recomendação de conteúdo para grupos específicos, automatizada de acordo com características do perfil do usuário e outras informações do contexto. Foi implementado 
o protótipo CA-Learning, para Web e para a plataforma Android. A partir dos dados de interação, foi possível recomendar conteúdo adicional ao já disponibilizado, de acordo com características dos grupos.

O e-Tutor [Santos et al. 2014] é um ambiente de aprendizagem interativo cujo objetivo é monitorar alunos e enviar notificações aos mediadores de um curso, a fim de facilitar ações de intervenção. Segundo os autores, esta ferramenta "atua diretamente sobre as atividades do aluno, monitorando o estado atual e gerando ações automáticas". Os resultados dos experimentos mostraram que, a partir da identificação de dificuldades, foram enviadas dicas ao aluno e mensagens de alerta ao tutor, a fim de que o mesmo pudesse decidir qual a melhor forma de intervenção.

\subsection{Técnicas de Visualização da Informação}

Neste trabalho foi usada uma técnica de visualização gráfica de informações conhecida como Treemap [Shneiderman 1992]. A área de visualização de informação é relativamente recente e busca aplicar técnicas de computação gráfica para ajudar no processo de análise de dados. Nela as informações são representadas por estruturas visuais gráficas manipuláveis, através de hierarquias de dados em espaços delimitados.

Johnson e Schneiderman (1991) propuseram a Treemap como técnica interativa que utiliza uma estrutura hierárquica para o espaço da tela a fim de representar os dados. Esse espaço bidimensional é preenchido por retângulos que representam as informações. A área destes retângulos é relativa e proporcional ao valor de uma variável previamente estabelecida, e assim a primeira área (espaço) vai se subdividindo para formar subáreas (ou subespaços) de dados. Esta técnica possui algumas vantagens que foram consideradas para sua utilização em nossa proposta, tais como: (i) flexibilidade; (ii) interatividade; (iii) usabilidade; (iv) interface simples; (v) fácil implementação; (vi) adaptável a AVAs.

A técnica Treemap tem sido utilizada ainda como apoio em avaliações e acompanhamento de atividades em AVAs, pois a ferramenta gráfica ajuda a melhorar a percepção do desempenho e comportamento dos estudantes. A possibilidade de se combinar variáveis e representá-las visualmente como um gráfico informativo fornece uma nova forma de extrair, interagir, manipular e analisar informações dos estudantes no ambiente virtual [Smith 2001].

Por este motivo, para nossa pesquisa foi escolhida esta técnica a fim de que se possa visualizar de forma gráfica as informações dos alunos em cursos do AVA Moodle e assim prover mais agilidade na análise das mesmas por parte dos tutores e professores.

\section{A arquitetura e funcionalidades do WebMonitor}

\subsection{Arquitetura}

A arquitetura do WebMonitor é mostrada na Figura 1. Pode-se resumir o fluxo de informações desta arquitetura nos seguintes passos:

1) Os estudantes acessam o AVA Moodle pelo desktop ou dispositivo móvel;

2) O Moodle registra as ações dos usuários nos $\log s$ do sistema (tabela mdl_log);

3) O mediador acessa o Web Monitor através do bloco instalado no Moodle; 
4) O WebMonitor recupera e processa os dados de $\log s$ da base de dados do Moodle;

5) A informação processada é transformada visualmente e disponibilizada ao mediador por meio de representações gráficas (Treemaps e gráficos de barras).

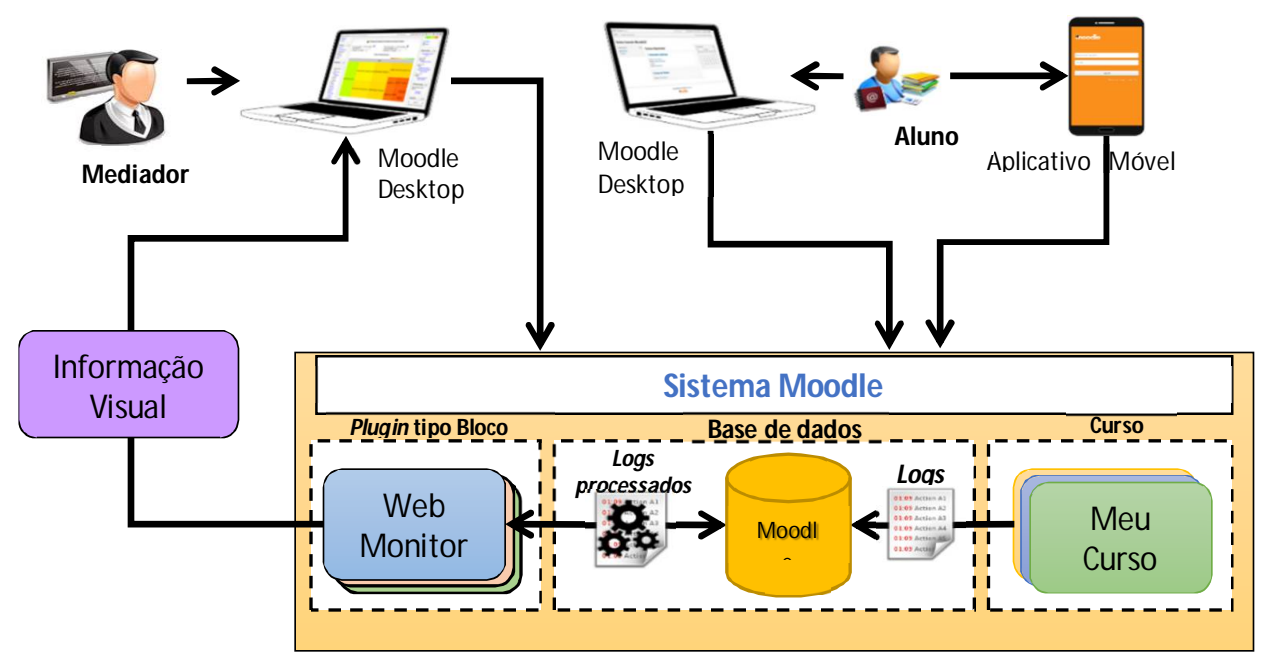

Figura 1. Arquitetura do WebMonitor

Toda a arquitetura do WebMonitor foi baseada no controle e monitoramento das interações, ou da ausência dessas no AVA Moodle, a fim que de auxiliar a identificação de estudantes com dificuldades na realização de suas atividades acadêmicas. Pois, o diagnóstico precoce de situações que possam evoluir para uma possível desistência ou reprovação pode ajudar no controle dos resultados finais na conclusão do curso [Teles Lucena et al. 2014].

\subsection{Descrição do aplicativo}

O aplicativo consiste de um plugin instalado no AVA Moodle, versão 2.5 (Moodle 2015). O WebMonitor foi desenvolvido na linguagem PHP5, e os gráficos utilizados para gerar a informação visual (Treemap e gráfico de barras) foram obtidos da biblioteca de APIs do Google [Developers Google 2015], ambas de uso livre. Visualmente, o plugin é um bloco localizado na parte superior à direita, da sala de aula virtual, conforme pode ser observado na Figura 2, assinalado por uma seta vermelha.

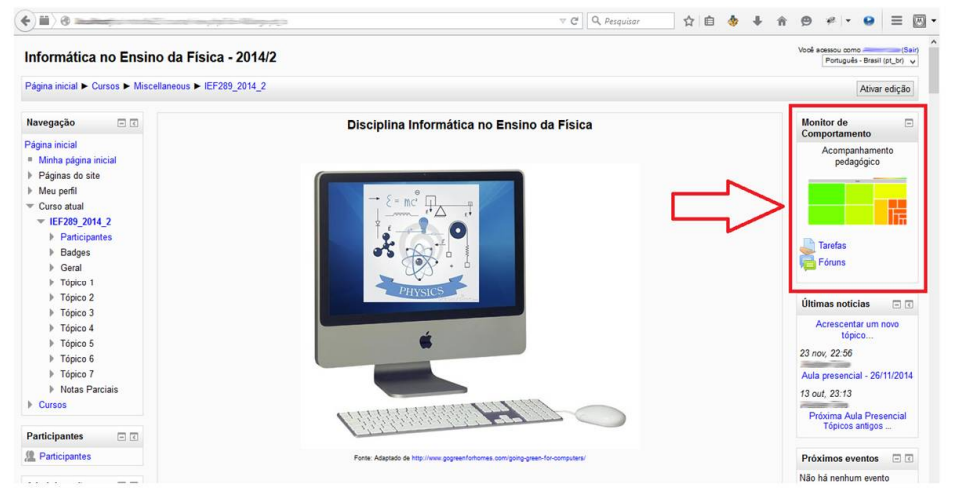

Figura 2. WebMonitor instalado em uma sala virtual do Moodle 
As interações que os alunos mantêm com os elementos de aprendizagem demonstram o grau de envolvimento do mesmo com o objeto a ser manipulado no processo de apropriação do saber. O Moodle apresenta os elementos de aprendizagem (EA) agrupados em duas categorias: Atividades e Recursos. As atividades são todos os objetos presentes no sistema que podem ser usados para estabelecer uma interação que exija uma postura ativa do usuário/estudante. Já os recursos são os elementos que podem ser utilizados como ferramentas didáticas para expor, explicar ou demonstrar um conteúdo ao usuário/estudante [Moodle 2015].

\subsection{Funcionalidades}

Ao ser inicializado, o plugin faz uma checagem no sistema para descobrir quais atividades e recursos estão sendo utilizados no ambiente virtual. Inicialmente foram desenvolvidas duas funcionalidades para acompanhamento de atividades: o monitoramento do envio de tarefas obrigatórias e a participação ativa em fóruns de discussão (Figura 3).

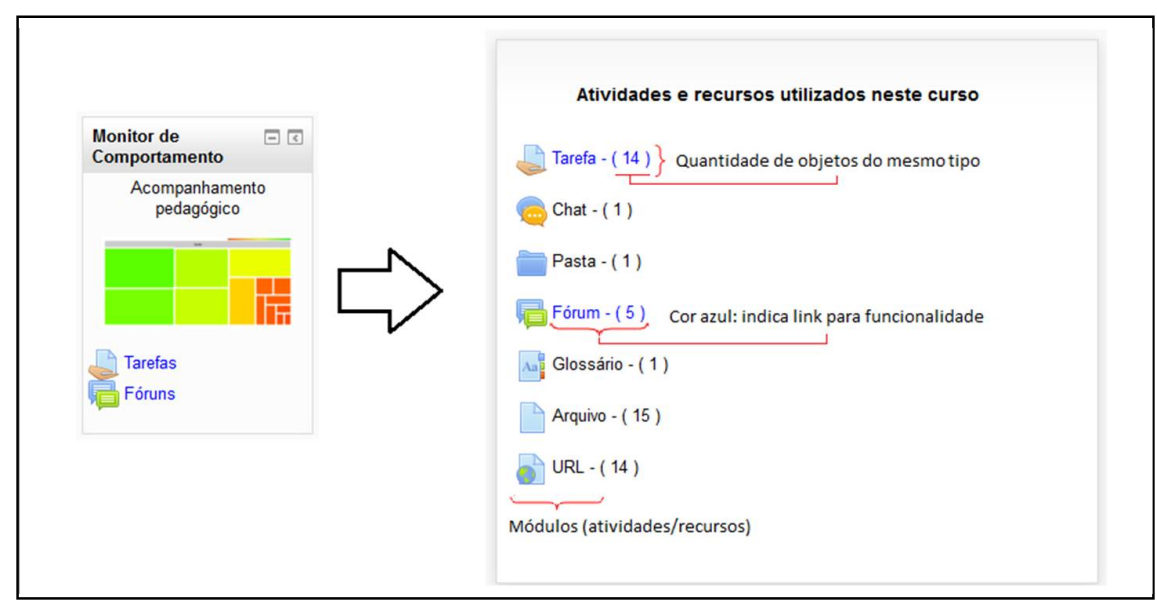

Figura 3. Bloco WebMonitor e painel de Atividades e Recursos

Para monitorar as tarefas, o plugin realiza uma busca no sistema por todas as atividades de entrega obrigatória. É disponibilizado aos mediadores um conjunto de seis funcionalidades que auxiliam no diagnóstico de alunos por níveis de participação. Os destaques enumerados podem ser observados na Figura 4.

- Destaque 1: Opção filtrar por grupo. Permite um controle detalhado da participação dos alunos, e que cada tutor monitore seu grupo em particular.

- Destaque 2: Percentual de estudantes por nível de participação. A área do gráfico é sensível ao clique e habilita a tela com opção para edição e envio de mensagem para os alunos do nível de participação escolhido.

- Destaque 3: Nível participação por aluno, através de gráfico Treemap, destacando as interações dos estudantes com os EA. O primeiro nível mostra o total de interações por módulos. No segundo nível é exibido o total de interações com um módulo específico e distribuídas por atividades. As cores variam de verde a vermelho, onde o verde indica um número maior de interações e vermelho o oposto. Caso haja uma distribuição igual das interações entre os EA, todos terão a cor amarelo, que indica o meio termo entre o verde e o vermelho (Figura 5). 
CBIE-LACLO 2015

Anais do XXVI Simpósio Brasileiro de Informática na Educação (SBIE 2015)

- Destaque 4: Resumo da participação do estudante na entrega das tarefas. O item 1 destacado serve como indicador da entrega de atividades, onde pode-se acompanhar a evolução do estudante. As tarefas entregues estão em cor verde. As não entregues, mas que ainda estão com prazo em aberto ficam na cor cinza, já as não entregues e com as datas de entrega vencidas são marcadas na cor vermelha.

- Destaque 5: Indicadores da participação dos estudantes. São usados para classificar os alunos de acordo com o percentual de tarefas entregues. Os círculos vermelhos indicam que a participação está entre 0 e $25 \%$ (zero e vinte e cinco por cento), e indica o nível de participação como muito baixo. Os círculos na cor laranja indicam nível de participação entre 26 a 50\% (vinte e seis e cinquenta por cento), este nível é considerado baixo, e assim como a classificação anterior, serve para alertar o mediador de uma possível situação de "risco". Os círculos na cor amarela indicam nível de participação entre 51 a 75\%, considerado grupo de risco no limite de uma participação mais efetiva. A quarta cor é a verde, e indica uma participação excelente em todas as atividades.

- Destaque 6: este item faz uma integração do plugin com a funcionalidade de envio de mensagem nativa do Moodle, desta forma facilita o processo de comunicação, permitindo o envio de mensagens individuais para os estudantes.

\section{Estudo de caso}

\subsection{Metodologia}

A metodologia seguida no estudo de caso com o WebMonitor foi planejada com o objetivo de orientar a realização da coleta de dados, e trata-se de uma pesquisa empírica, de caráter exploratório [Yin 2010], pois visa identificar o comportamento e hábitos de estudantes no uso de AVA utilizando uma ferramenta de suporte a atividades no ensino mediado por tecnologias. Dessa forma, a metodologia aplicada no experimento consistiu em: (i) estabelecimento do objeto para o estudo de caso; (ii) descrição dos procedimentos de testes; (iii) coleta e análise de dados.

\subsection{Experimentos e resultados}

O cenário escolhido para estudo de caso consistiu em três turmas de graduação da disciplina Informática no Ensino da Física, do curso de Licenciatura em Física, da Universidade Federal do Amazonas, oferecida na modalidade semipresencial. As turmas foram ministradas nos períodos 2014/1 (Turma 1), 2014/2 (Turma 2) e 2015/1 (Turma 3) e em cada uma estavam matriculados de 8 a 10 alunos. Todas as turmas foram gerenciadas pelo AVA Moodle e somente a Turma 3 teve acesso também por dispositivos móveis.

Como indicadores quantitativos foram utilizadas as interações dos alunos no ambiente virtual e o uso do aplicativo móvel. As Tabelas 1,2 exibem estas informações de acordo com a categoria dos EA acessados. 
CBIE-LACLO 2015

Anais do XXVI Simpósio Brasileiro de Informática na Educação (SBIE 2015)

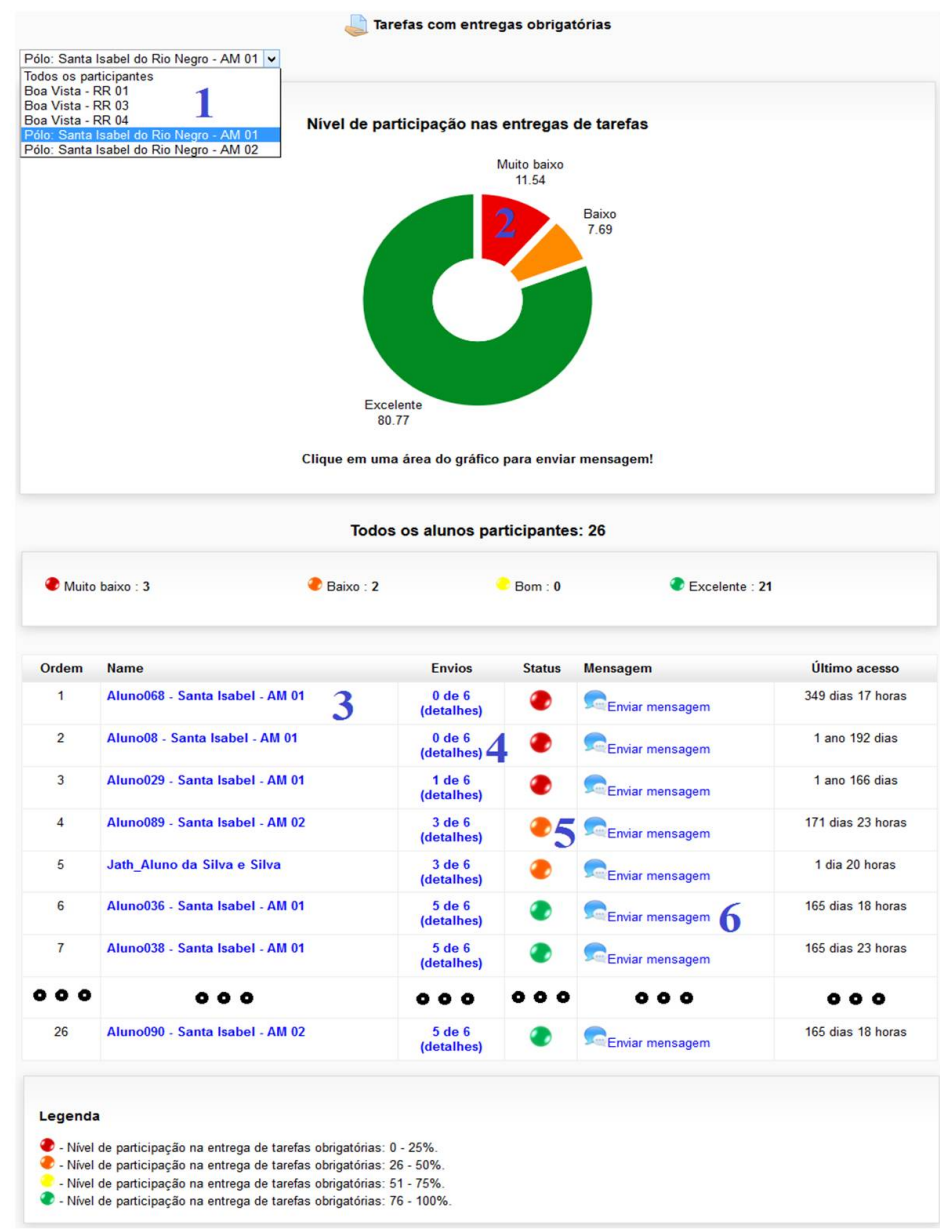

Figura 4. Monitoramento de tarefas

A Tabela 3 mostra os dados de acesso por aluno acessibilidade ao AVA (convencional ou móvel). Os mediadores da turma utilizaram os gráficos Treemap para acompanhar o desempenho dos alunos em cada atividade e puderam intervir com mensagens de alerta quando a interação número de interações estava abaixo da média.

Tabela 1 - Interações por categoria de EA - Turma 1

\begin{tabular}{|c|c|c|}
\hline Categoria objeto & № de instâncias & № Interações \\
\hline Atividade & 17 & 874 \\
\hline Recurso & 52 & 383 \\
\hline Total & 69 & 1257 \\
\hline
\end{tabular}

Tabela 2 - Interações por categoria de EA - Turma 2

\begin{tabular}{|c|c|c|}
\hline Categoria objeto & № de instâncias & № Interações \\
\hline Atividade & 17 & 607 \\
\hline Recurso & 52 & 233 \\
\hline Total & 69 & $\mathbf{8 4 0}$ \\
\hline
\end{tabular}




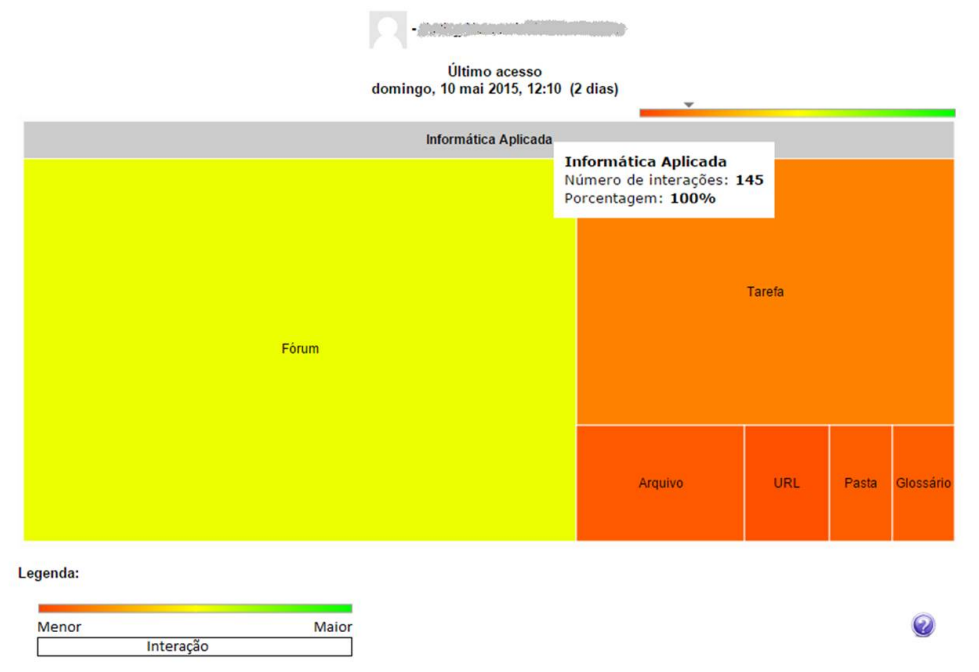

Figura 5 -Treemap das interações de um aluno na visão inicial

Tabela 3 - Interações pelo modo de acesso - Turma 3

\begin{tabular}{|l|c|c|c|c|}
\hline Aluno & Web & Smartphone & Tablet & Total de Interações \\
\hline Aluno 1 & 0 & 0 & 0 & 0 \\
\hline Aluno 2 & 0 & 0 & 0 & 0 \\
\hline Aluno 3 & 7 & 0 & 0 & 7 \\
\hline Aluno 4 & 27 & 0 & 0 & 27 \\
\hline Aluno 5 & 33 & 5 & 0 & 38 \\
\hline Aluno 6 & 53 & 0 & 0 & 53 \\
\hline Aluno 7 & 45 & 16 & 0 & 61 \\
\hline Aluno 8 & 59 & 2 & 4 & 65 \\
\hline Aluno 9 & 60 & 17 & 0 & 77 \\
\hline Aluno 10 & 73 & 10 & 0 & 83 \\
\hline Aluno 11 & 122 & 21 & 0 & 143 \\
\hline Aluno 12 & 153 & 10 & 0 & 163 \\
\hline Média & $\mathbf{5 2 , 6 7}$ & $\mathbf{6 , 7 5}$ & $\mathbf{0 , 3 3}$ & $\mathbf{5 9 , 7 5}$ \\
\hline
\end{tabular}

\section{Análise e Discussões}

A quantidade de interações que cada estudante estabelece com os elementos no AVA pode representar o comportamento do mesmo. Como não foi estabelecido nenhum limite de quantidades de acessos ou interações por elementos, a observação desse indicador pode ajudar na identificação de algum comportamento "de risco" quando este está situado fora do padrão (média) de interações.

O indicador de interações pode ficar fora da média de acesso em duas situações opostas: pelo número reduzido ou pela quantidade demasiada de interações estabelecidas com um ou mais elementos do AVA. No primeiro caso, a baixa interação com os elementos do ambiente pode comprometer o processo da aprendizagem pela falta de contato com conteúdo disponibilizado através desses elementos. Já no segundo caso, um número de interações elevado com um ou mais recursos pode revelar indícios de que o 
aluno esteja passando por dificuldades para manipular esses elementos no AVA. Em uma análise prévia dos dados, foi possível estabelecer a correspondência entre os índices de evasão e reprovação nas turmas estudadas e a média de interações em atividades do curso. Uma outra informação a se considerar é que o índice de desistência na Turma 3, a única a usar o aplicativo móvel, foi o menor entre todas estudadas. É certo que ainda não se pode concluir que a utilização deste aplicativo motivou os alunos, mas mostra-se como um ponto de observação e experimento com futuras turmas.

Contudo, deve-se usar esses indicadores com cautela, sobretudo observando as notas correspondentes às tarefas, pois as ferramentas de visualização determinam se as tarefas são entregues ou não, é até o momento não foi desenvolvido um mecanismo para se considerar o peso de cada tarefa. A análise do desempenho de uma turma ou do aluno individualmente ainda é subjetiva e está a cargo do mediador da turma.

\section{Conclusão}

Através da estratégia de um estudo de caso exploratório que utiliza uma ferramenta visual de acompanhamento e monitoramento de atividades e contando com o suporte de um aplicativo para dispositivos móveis, este artigo oferece informações para uma análise mais profunda sobre a relação entre a quantidade de acesso a atividades da sala de uma aula virtual e os índices de reprovação ou desistência em cursos à distância ou semipresencial. No estudo destes dados buscou-se identificar quais elementos contidos nos mesmos poderiam caracterizar o comportamento do aluno em um AVA.

O WebMonitor apresentou-se com uma ferramenta de suporte ao gerenciamento de desempenho em um AVA, através do monitoramento da participação dos estudantes em tarefas avaliativas. Pois, as informações coletadas pelo sistema ofereceram um levantamento preciso da participação discente em atividades específicas. A partir destes dados foi possível identificar a possibilidade de ocorrência de evasão ou reprovação, e ainda fornecer subsídios para uma intervenção direta a fim de se evitar estes problemas. Além disso, este aplicativo ofereceu como inovação para professores e alunos a proposta de um acompanhamento intuitivo de atividades, devido ao uso de técnicas de visualização gráfica de informações.

Como propostas futuras, sugere-se expandir os experimentos com o aplicativo móvel e disponibilizar a ferramenta para outros AVAs. O uso de agentes inteligentes também deveria ser considerado como opção para prover ao sistema WebMonitor uma autonomia no acompanhamento e monitoramento, a fim de alertar os responsáveis pela turma sobre comportamentos individuais de risco ainda não detectados. Uma extensão dos estudos enfocando a análise comparativa de desempenho acadêmico, além de uma avaliação do impacto do aplicativo nas turmas que o utilizaram também auxiliaria no enriquecimento deste trabalho.

\section{Agradecimentos}

Os autores agradecem o apoio financeiro da Fundação de Amparo à Pesquisa do Estado do Amazonas (FAPEAM) através do Edital N 016/2013, Projeto PROTI-Pesquisa sob o processo $\mathrm{N}^{\circ}$ 062.00597/2014. Além disso, parte dos resultados apresentados neste trabalho foi obtida através de atividades de P\&D do "Programa de Qualificação em Grande Escala em Tecnologias Móveis - PROMOBILE", projeto patrocinado pela Samsung Eletrônica da Amazônia Ltda. nos termos da lei federal brasileira nº 8.248/91. 


\section{Referências}

Almeida, A.; Pimentel, E. P.; Stiubiener, I. (2012) "Estratégias para o Monitoramento de Ações de Tutoria na Educação a Distância”. In: Anais do I Congresso Brasileiro de Informática na Educação.

Developers Google. (2015) "Biblioteca gratuita para desenvolvedores". https://developers.google.com/chart/?csw=1. Acessado em 15/05/2015.

Ferreira, V. da S. e Elia, M. da F. (2013) "Uma modelagem conceitual para apoiar a identificação das causas da evasão escolar em EAD”. In: II Congresso Brasileiro de Informática na Educação, Campinas.

Johnson, B. and Shneiderman, B. (1991) "TreeMaps: A space - filling approach to the visualization of hierarchical information structures". Proceedings of IEEE Visualization. San Diego. p. 284 - 291.

Moodle. (2015) "Modular Object-Oriented Dynamic Learning Environment". http://moodle.org/. Acessado em 15/05/2015.

Oliveira, E. H. T., Teles Lucena, K. K., Nozawa, E. H., Lucena, W. (2012) "Distance Education with remote poles: an example from the Amazon region". In: Frontiers in Education (FIE) 2012. Seattle, WA.

Pereira, A. C. (org.). (2007) "Ambientes Virtuais de Aprendizagem - em diferentes contextos”. Editora Ciência Moderna, Rio de Janeiro.

Reis, G., Barrére, E. (2014) "Recomendação Colaborativa de Conteúdos Educacionais para Dispositivos Portáteis". In: XXV Simpósio Brasileiro de Informática na Educação, Dourados. In: Anais do III Congresso Brasileiro de Informática na Educação.p. 934-943.

Santos, R. S., Luz, B. N., Martins, V. F., Guimarães, M. P. (2014) “eTutor: Um Ambiente de Aprendizagem Interativo". In: XXV Simpósio Brasileiro de Informática na Educação, Dourados. In: Anais do III Congresso Brasileiro de Informática na Educação. p. 476-485.

Shneiderman, B. (1992) "Tree visualization with treemaps: A 2-D space-filling approach”. ACM Transactions on Graphics 11(1), p. 92-99.

Smith, A. and Fiore, T. (2001) "Visualization Components for Persistent Conversations". In: Beaudouin-Lafon, Michel and Jacob, Robert J. K. (eds.) Proceedings of the ACM CHI 2001 Human Factors in Computing Systems Conference, Seattle, Washington.

Teles Lucena, K. K., Silva, J. S., Frota, V., Oliveira, E. H. T., Gadelha, B. F. (2014) "MobiMonitor: a Mobile App for Monitoring Distance Courses in the Amazon Region". In: IEEE Frontiers in Education Conference, 2014, Madrid. IEEE Frontiers in Education Conference Proceedings, 2014. v. 1. p. 2126-2133.

Yin, R. K. (2010) “Estudo de caso: planejamento e métodos”. 2a edição. Bookman, Porto Alegre. 